\title{
INTELLIGENCE DES ANIMAUX : LA RÉPONSE DÉPEND DE LA QUESTION
}

\author{
Vinciane Despret
}

Editions Esprit | Esprit

\author{
2010/6 - Juin \\ pages 142 à 154
}

\section{ISSN 0014-0759}

Article disponible en ligne à l'adresse:

http://www.cairn.info/revue-esprit-2010-6-page-142.htm

Pour citer cet article :

Despret Vinciane, «Intelligence des animaux : la réponse dépend de la question », Esprit, 2010/6 Juin, p. 142-154. DOI : 10.3917/espri.1006.0142

Distribution électronique Cairn.info pour Editions Esprit.

(C) Editions Esprit. Tous droits réservés pour tous pays.

La reproduction ou représentation de cet article, notamment par photocopie, n'est autorisée que dans les limites des conditions générales d'utilisation du site ou, le cas échéant, des conditions générales de la licence souscrite par votre établissement. Toute autre reproduction ou représentation, en tout ou partie, sous quelque forme et de quelque manière que ce soit, est interdite sauf accord préalable et écrit de l'éditeur, en dehors des cas prévus par la législation en vigueur en France. II est précisé que son stockage dans une base de données est également interdit. 


\title{
Intelligence des animaux : la réponse dépend de la question
}

\author{
Vinciane Despret ${ }^{*}$
}

$\mathbf{O}_{\mathrm{N}}$ a longtemps pensé que les perroquets ne parlaient pas. Si ce n'est, bien entendu, qu'ils parlaient «comme des perroquets », autant dire sans la condition qui consacre l'accès au langage: savoir ce qu'on dit. L'expression est à ce point familière qu'on pourrait penser qu'elle se fonde sur une réalité commune. Mais ne dit-on pas aussi que les cochons sont «sales comme des cochons»? Pour ceux qui les connaissent un peu, les cochons semblent attacher au contraire une grande importance à leur toilette corporelle. Toutefois, ils utilisent, pour ce faire, d'autres matériaux que nous. De mauvaises réputations, relativement mal fondées, se créent, se propagent et font rumeur. Car les perroquets parlent, pour ceux qui connaissent vraiment les perroquets, il n'y a aucun doute là-dessus. Alors la question qui s'impose apparaît toute différente et consiste à se demander pourquoi certains continuent à penser qu'ils ne le peuvent pas.

La philosophe Vicki Hearne, qui est aussi dresseuse de chiens et de chevaux, propose une réponse à cette question pour ce qui concerne ses collègues philosophes qui continuent à propager de telles rumeurs. Certes, concède-t-elle, on pourrait avancer que les philosophes manifestent parfois de sérieux problèmes de rivalité en ce qui concerne le langage ${ }^{1}$. On pourrait même, si l'on exige des preuves de cette accusation, la suivre encore et détourner quelque peu l'affirmation de Wittgenstein lorsqu'il suggère que même si un lion pouvait parler, nous ne pourrions le comprendre ${ }^{2}$. Le problème

* Psychologue et philosophe, maître de conférences à l'université de Liège, elle a publié notamment Quand le loup habitera avec l'agneau, Paris, Les Empêcheurs de penser en rond, 2001 et Hans, le cheval qui savait compter, Paris, Les Empêcheurs de penser en rond, 2004.

1. Vicki Hearne, Animal Happiness, New York, Harper \& Collins, 1994, p. 3.

2.Ibid., p. 168. 
pourrait, de fait, être défini de cette manière: ce sont les philosophes qui ne comprennent pas les perroquets. Hearne nous en propose l'expérience. Imaginez qu'un philosophe se trouve face à un perroquet. Si notre philosophe a quelque goût pour l'empirisme et les mises à l'épreuve, il y a de fortes chances que la rencontre se déroule comme suit: le philosophe va poliment demander au supposé bavard volatile comment il se sent aujourd'hui. Et le perroquet lui répondra quelque chose comme «des nuages roses encombrent le ciel». À moins d'envisager de donner une interprétation audacieusement métaphorique à ce qui relèverait alors d'un constat d'humeur, ce que le philosophe, à raison, ne fera sans doute pas, le malentendu ne peut que s'installer. N'adoptez toutefois pas précipitamment l'hypothèse qui s'impose au philosophe et selon laquelle le perroquet viendrait de démontrer, malgré la chance qui lui était offerte, qu’il ne comprend pas ni ce qu'on lui dit ni ce que lui-même profère. Il s'agit de bien autre chose. En fait, selon Hearne, les perroquets ne supportent pas de laisser quelqu'un d'autre prendre le contrôle du sujet de la conversation, même si c'est un philosophe intéressé et poli. Dès lors, la rumeur selon laquelle les perroquets n'ont aucune compétence hormis celle d'une imitation sans intelligence, continue à se propager. Car le philosophe ne pourra jamais imaginer que le perroquet, comme lui, s'imagine qu'il est impensable de laisser à un autre le choix de ce dont il est important de parler.

Il y a heureusement quelques exceptions chez les philosophes. Et le fait qu'Élisabeth de Fontenay ait donné à l'un de ses livres le magnifique titre le Silence des bêtes et qu'elle l'ait prolongé, comme une possible réponse, par Traduire le parler des bêtes nous dit bien de la volonté de ceux qui ont pris le problème au sérieux: ce silence n'existe que dans un monde de sourds, ou encore dans un monde assourdissant de bavardages humains, incapables de ralentir, le temps d'entendre les animaux parler et surtout de les entendre nous répondre. «Fallait-il, conclut la philosophe aux dernières lignes de cet essai, qu'il ne fût pas écrivain, et pas traducteur, ce Wittgenstein pour énoncer que si, des bêtes pouvaient parler, on ne pourrait pas les comprendre ${ }^{3}$ !»

Puisqu'il s'agit de ralentir, ralentissons, et arrêtons-nous quelques instants à ce livre. Ce qu'y concocte la philosophe comme dans une cuisine de bénéfices - comment dire autrement le contraire des maléfices? -, c'est la possibilité des bonnes traductions: chercher les amis, bien rares, un peu moins chez les anciens dit-elle, parmi lesquels on trouve encore quelques-uns ayant consenti à l'idée d'une possible communauté avec les bêtes. Il y aurait eu tout au long de

3. Élisabeth de Fontenay et M.-C. Pasquier, Traduire le parler des bêtes, Paris, L'Herne, 2008 , p. 36. 
notre histoire littéraire, plus de faux amis que de vrais. Du côté des faux amis, on trouvera tous ceux, dit elle, qui font parler les animaux, «à la place des animaux »: de bêtes, dans ces circonstances, il n'en est pas vraiment question; elles n'y sont que figures, tropes ou prêtenom ${ }^{4}$. Du côté des autres, on trouvera ceux qui parlent d'elles, et qui «n'essaient pas tant de les faire venir à nous que d'aller à elles ${ }^{5}$ » .

Certes, l'anecdote qui ouvrait cet article conduit encore à une troisième voie, celle que m'inspirent les travaux de Dominique Lestel lorsqu'il désigne les laboratoires où des non-parlants sont invités à entrer dans une part de notre langage comme des lieux de convivialité où s'épanouissent les relations de communautés hybrides : il ne s'agit plus tant de parler de ou de parler pour, mais de parler avec ${ }^{6}$. Le «faire parler» prend alors une tout autre signification que celle que lui attribuait Élisabeth de Fontenay et rejoint les pratiques des vrais amis : on y «parle de» mais ensemble.

Ce «parler de» ensemble, l'anecdote du perroquet en témoignait clairement, s'accompagne d'une complication que ne rencontrent pas les deux autres postures; les risques n'y sont pas les mêmes. Avec la première, celle par laquelle on «fait parler» mais dans un monologue, on ne risque jamais de se tromper, et pour cause, on sait de quoi, ou de qui on parle; avec la seconde, tout ce qu'on risque, c'est de ne pas savoir que l'on se trompe. En revanche, dans la situation du «parler ensemble», l'échec est immédiatement perceptible. Ce que le philosophe expérimente avec le perroquet qui parle des nuages relève de cette complication; le perroquet restera incompréhensible, non pas pour donner raison à Wittgenstein, mais parce qu'il faut, pour le comprendre, en passer par la manière dont lui-même comprend la situation qui lui est proposée.

Le témoignage qui clôt le livre d'Élisabeth de Fontenay n'a, à cet égard, rien d'innocent: elle cite un long texte que Marguerite Duras a consacré à Koko, la petite gorille ayant appris la langue des signes. Ce texte parle d'une anecdote, une anecdote qui bouleverse l'auteure: certains matins, à son lever, Koko affirme qu'elle est triste, et qu'elle ne sait pas pourquoi. Et ce «je ne sais pourquoi », loin de renvoyer Koko à un quelconque manque de réflexivité, au contraire, construit avec elle une histoire commune, dans laquelle l'expérience

4. Voir à cet égard un constat similaire, quoique encore plus sévère et plus injustement sévère chez Jacques Derrida (L'animal que donc je suis, Paris, Gallilée, 1997). Pour une critique de cet «injustement sévère», je renvoie à Donna Haraway, When Species Meet, Minneapolis, University of Minnesota Press, 2008.

5. É. de Fontenay et M.-C. Pasquier, Traduire le parler des bêtes, op. cit., p. 29.

6. Dominique Lestel, l'Animalité. Essai sur le statut de l'humain, Paris, Hatier, coll. "Optiques », 1996; "How Chimpanzees have Domesticated Humans", Anthropology Today 14, 1998, 3, p. 12-15; voir également, dont ce présent texte pourrait constituer une proposition de prolongement en suivant, à ma mode, les chemins si intéressants qu'il emprunte aujourd'hui, l'Animal singulier, Paris, Le Seuil, 2004. 
d'une petite gorille vient rejoindre la nôtre, celle de la mélancolie. Nous aussi, nous avons des chagrins en deçà de tout savoir.

Certes, peu de choses semblent communes entre cette histoire de perroquet mettant un philosophe en bouteille et la tristesse mélancolique que partagent une gorille et une écrivain. Mais elles relèvent toutes deux d'un même geste, celui de Hearne, celui de Duras : toutes deux s'inquiètent de la manière dont l'animal fait l'expérience de la situation. Ces deux animaux surprennent; ils ne sont pas là où on les attendait.

\section{Qu'est-ce que poser une bonne question?}

Je ne néglige cependant pas le fait que l'hypothèse de Hearne ne puisse convaincre tout le monde. Celle de la psychologue Irène Pepperberg pourrait sans doute répondre mieux à nos habitudes de pensée tout en se signalant, à mon sens, comme une de ses traductions possibles. Pour Pepperberg, si les perroquets ne parlent pas, c'est que cela ne les intéresse pas. Et l'échec des chercheurs tient à cette simple hypothèse: ils n’ont pas réussi à comprendre ce qui, dans le langage, pouvait bien intéresser les perroquets. Comme vous le voyez, on n'est pas tellement loin de Hearne. Pepperberg raconte, pour caractériser ces échecs, qu'un behavioriste avait réussi à apprendre à un perroquet à dire «hello » chaque fois qu'il entrait dans le laboratoire. Mais ce succès s'avéra finalement bien éphémère, puisque le perroquet se mit à tonitruer «hello» dans des circonstances les plus diverses et toutes plus inappropriées les unes que les autres. Le chercheur n'avait pas pensé que le fait de récompenser, comme le veut la méthode du conditionnement, chaque salutation par une cacahuète, avait fini par induire chez le perroquet un tout autre usage sémantique du terme «hello» et que ce dernier en était venu à signifier cacahuète. Bref, à l'issue de ce type de recherches, les behavioristes en ont conclu que les perroquets ne peuvent pas parler, au grand étonnement d'ailleurs des dresseurs et des propriétaires de perroquet. Mais ces behavioristes n'ont jamais imaginé qu'eux-mêmes avaient conduit les perroquets à l'incompétence. En définissant aussi chichement ce qui intéresse un perroquet, des cacahuètes, ils ont obtenu une réponse à la hauteur de cet intérêt: une demande constante de cacahuètes. Il n'y avait visiblement pas grand-chose d'autre à demander.

Ils n'ont surtout pas pu invoquer une hypothèse qui, pourtant, aujourd'hui s'impose: ce que les animaux font au laboratoire, à la demande des scientifiques, traduit d'abord et avant tout une opinion, un jugement sur ce qui leur est proposé. Le psychologue Crespi, dans les années 1940, a magnifiquement mis cette hypothèse à l'épreuve, mais je crains qu'elle n'ait pas apporté toutes les leçons qu'on aurait pu en tirer. Crespi montre en effet que si l'on donne à des rats, au 
cours d'un apprentissage, une récompense beaucoup plus importante qu'aux essais précédents, ceux-ci augmentent leur régime d'activité juste après cette offrande aussi arbitraire qu'inopinée. Le groupe auquel est donnée d'office cette récompense importante à tous les essais ne manifeste pas les mêmes performances. Si on teste la procédure inverse, en diminuant arbitrairement la récompense, Crespi envisage de traduire la réaction des rats par la dépression, ce que certains chercheurs aujourd'hui me proposent de traduire par « déception».

Si l'on peut soutenir cette hypothèse selon laquelle les animaux jugent de ce qui leur est proposé, et répondent en fait à cette question plutôt qu'à celle qui leur est posée, on doit alors considérer plus sérieusement que les animaux répondent à ce qu'ils perçoivent, ou comprennent ou imaginent de nos intentions. Cette possibilité de non seulement prêter des intentions aux congénères, mais de nous les accorder également (la réciproque, remarquons-le en passant, a longtemps tardé) a été maintes fois expérimentée au cours de ces dernières années. En 1978, David Premack et G. Woodruff vont donner une nouvelle orientation à leurs recherches avec les chimpanzés. On avait jusqu'alors interrogé des chimpanzés physiciens, expliquent-ils, puisqu'on leur demandait de résoudre des problèmes comme ceux d'attraper une banane avec un bâton, un tabouret et une caisse; nous nous sommes maintenant attelés à interroger des chimpanzés psychologues: auraient-ils une théorie de l'esprit? Si l'expérimentateur cherche une friandise dont le chimpanzé connaît la cachette, ce dernier généralement l'aide. Mais si l'humain refuse de lui offrir, on constatera qu'à l'essai suivant, le chimpanzé finit par lui mentir. Certes, on pourrait toujours réduire l'explication à l'hypothèse du conditionnement: les chimpanzés ne feraient qu'obéir à la règle des associations apprises. Les deux auteurs ne le pensent pas. Nous prêtons spontanément des intentions aux autres parce que c'est plus simple comme explication, disent-ils, le singe fait probablement de même: «Le singe pourrait s'avérer n'être que mentaliste. À moins que nous nous trompions lourdement, il n'est pas assez intelligent pour être behavioriste ${ }^{7}$.»

Par ailleurs, des expériences similaires ont été proposées aux corbeaux. Les corbeaux ont pour habitude de cacher de la nourriture. Ils le font différemment s'ils se sentent observés et peuvent même prétendre la cacher, et quand les autres se mettent à la chercher, la dissimuler réellement en profitant de leur inattention ${ }^{8}$. Plus étonnant, les corbeaux sembleraient jouer à cacher des objets. Or, si un obser-

7. D. Premack et G. Wooldruff, "Does the Chimpanzee have a Theory of Mind?", The Behavioral and Brain Science, 1978, 4, p.516-526, p. 526. Je remercie mon étudiant Thibaut De Meyer d'avoir attiré mon attention sur cet article.

8. Bernd Heinrich, Mind of the Raven, New York, Harper \& Collins, 2000. 
vateur humain vole un de ces jouets cachés dans le contexte du jeu, on remarquera un changement radical d'attitude vis-à-vis de cette personne précise lorsqu'il s'agira de nourriture: le corbeau prendra bien plus de précautions, veillera à être en dehors de son champ de vision et prendra plus de temps à la recouvrir que s'il est en présence d'une personne inconnue'.

Les exemples de ce type ont commencé, ces derniers temps et sous l'effet salutaire des théories cognitives, à abonder. Si les animaux modifient leurs attitudes en fonction des intentions qu'ils semblent nous prêter, dans ces expériences qui les interrogent sur cette question, il me semble alors évident qu'ils ne cessent de le faire, dans toutes les expériences, quelle que soit la question qu'on leur pose.

Mais en acceptant cette hypothèse, on se doit alors d'en assumer une autre; toute situation expérimentale serait, d'entrée de jeu, marquée par l'artefact. L'artefact, je le définirais très simplement, consiste dans le fait que l'animal semble répondre à la question qui lui est adressée alors qu'en fait, il répond à une tout autre question, une question dont le chercheur lui-même ignore qu'elle a été posée.

Or, il me semble que c'est exactement la possibilité d'avoir pris cet artefact en compte, non pour le contourner, mais pour l'utiliser activement comme une ressource, qui explique le succès de Pepperberg avec son perroquet Alex. Car là où toutes les expériences de conditionnement des behavioristes avaient échoué, la psychologue Irène Pepperberg a accompli, avec Alex, le bel événement de la conversation interspécifique ${ }^{10}$.

\section{Un dispositif qui n'annule pas l'observation}

Certes, le dispositif lui-même tendrait à nous faire penser le contraire de mon hypothèse, celle selon laquelle la psychologue prend activement en compte la possibilité de créer un artefact, puisque Pepperberg, justement, va cacher à Alex sa véritable intention de le faire parler. On pourrait en effet penser que nous avons là affaire à des leurres semblables à ceux qu'on utilise avec les sujets humains quand on leur cache le réel enjeu de l'expérience, afin de veiller à éradiquer l'artefact par excellence: que le sujet ne fasse ce qu'il fait que parce que l'expérimentateur le lui a demandé. Ceci pourrait choquer le bon sens: bien sûr que le sujet fait ce qu'il fait parce qu'on le lui a demandé, comment pourrait-il en être autrement?

9. Thomas Bugnyar et Bernd Heinrich, "Ravens, Corvux Corax, Differenciate Between Knowledgeable and Ignorant Competitors", Proceedings of the Royal Society B, août 2005, vol. 272, $\mathrm{n}^{\circ} 1573$, p. 1641-1646. Je remercie mon étudiante Ariane D'Hoop pour m'avoir appris les choses les plus passionnantes sur l'effet d'audience.

10. I. Pepperberg, "Grey Parrots Intelligence", Proceedings of the International Aviculturists Society, janvier 1995, p. 11-15. 
Cependant, ce n'est pas avec le bon sens qu'il faut aborder ces sujets. Les choses sont un peu plus compliquées ${ }^{11}$. Supposons qu'un expérimentateur veuille étudier la colère. S’il demande à ses sujets de se mettre en colère, en pensant par exemple à un événement susceptible de rappeler et de réactiver ce sentiment, il ne pourra pas prétendre avoir réellement affaire à une colère spontanée, mais sera suspecté d'avoir créé, chez son sujet, une colère de «complaisance ». Dès lors, l'expérimentateur va créer une tout autre situation: il va annoncer à ses sujets qu'il s'agit d'accomplir une épreuve d'adresse en effectuant une tâche difficile, par exemple lancer des anneaux de bois autour de cibles $^{12}$. Or, cette épreuve s'avère en fait impossible. Le sujet va se décourager, le scientifique insister. Au bout d'un temps assez long, et quelques provocations de la part du scientifique, le sujet se met en colère. On a la réponse spontanée. Il ne s'est pas mis en colère parce qu'on le lui a demandé. Le psychologue pourra alors prétendre à ce que Martin Orne appelle la validité écologique de son expérience: ce qui s'est passé au laboratoire peut expliquer ce qui se passe dans la vie réelle. Comme s'il n'y avait pas eu de laboratoire.

Retour à Pepperberg: elle va également, à l'instar des psychologues travaillant avec les humains, masquer ses intentions. Mais il ne s'agit pas cette fois de contrer la «complaisance» de son sujet - seuls les humains sont sensibles à l'autorité des scientifiques et au salut de la science -, au contraire, il s'agit plutôt de travailler avec la récalcitrance du perroquet. La ruse est en fait bien plus intéressante qu'un leurre destiné à masquer des intentions. Le problème était le suivant: comment dépasser le stade de la cacahuète? Pepperberg est allée interroger ceux qui ont réussi à faire parler les perroquets, leurs dresseurs. Ces derniers lui ont signalé une caractéristique intéressante de leurs animaux - une caractéristique qui explique sans doute encore plus clairement le désastre relationnel que constitue la rencontre d'un perroquet et d'un philosophe sous un ciel de nuages roses: les perroquets ont un sens aigu de la rivalité. Une situation d'apprentissage qui ne représente pas un défi face à un tiers n’a aucune chance de les intéresser.

11. On notera qu'une bonne part des expériences menées avec des sujets humains, en psychologie, s'est construite pour répondre à ce problème. C'est d'ailleurs la raison pour laquelle les expérimentateurs ont fabulé à l'envi sur les véritables enjeux et buts de l'expérience (jusqu'à ce que l'exigence du consentement éclairé s'impose et rende les leurres de plus en plus difficiles), et donc se sont efforcés de cacher à leurs sujets leurs réelles intentions, de peur que ceux-ci ne répondent, souvent favorablement, à cette intention et non au problème dont ils sont censés témoigner. Le fait que les sujets ignorent réellement les intentions du chercheur a été, à de multiples reprises, remis très sérieusement en question. Voir à cet égard V. Despret, Hans, le cheval qui savait compter, Paris, Les Empêcheurs de penser en rond, 2004.

12. Cette expérience a été réalisée par Tamara Dembo en 1925. Elle sera publiée en 1931. On la trouve mentionnée dans l'Esquisse d'une théorie des émotions, de Sartre, qui lui-même l'a trouvée résumée dans le travail de Paul Guillaume, Psychologie de la forme. Je me suis servie de la traduction anglaise de l'article de Dembo, "The Dynamic of Anger", dans Joseph De Rivera (ed.), Field Theory as Human Science, New York, Gardner Press, 1976, p. 324-422. 
Aussi, la chercheuse va-t-elle modifier le dispositif habituel de l'expérimentation. On ne demande rien au perroquet si ce n'est d'assister à la leçon qu'un chercheur donne à un de ses collègues complices. Il arrive toujours un moment où, si le perroquet présente cette heureuse faiblesse spécifique, il voudra devancer l'élève et répondre à sa place. Le processus est lancé, ne reste qu'à convaincre l'animal de laisser l'expérimentateur lui imposer le choix du thème. Pour s'assurer de sa meilleure collaboration, on lui laisse choisir la récompense, il n'a qu'à la demander: promenade, friandise, jouet. Parler est devenu un enjeu intéressant pour l'animal puisque cela lui permet d'agir sur le monde et sur les autres et de leur faire faire des choses. Cela change des dispositifs où l'on affame les sujets pour les motiver par le plus simple et le moins imaginatif des renforcements. Qu'un perroquet se taise dans une expérience ne peut plus dès lors conduire à la conclusion qu'il ne peut apprendre à parler; cela peut signifier, beaucoup plus simplement, qu'il exprime une opinion sur ce qu'on lui demande et sur la manière dont on lui demande: il n'y a rien à dire. C'est d'ailleurs de cette manière que Pepperberg conclut son succès avec Alex. Mon expérience, explique-t-elle, ne permet en rien d'affirmer que tous les perroquets peuvent parler, ni que les perroquets du Gabon peuvent le faire. Elle indique seulement que, en parlant, Alex juge de la pertinence du dispositif qui lui est proposé.

Ce qui veut dire que la généralisation change de portée: on ne généralise plus sur les êtres - ce qui vaut pour Alex ne vaut que pour Alex -, on généralise à présent sur les dispositifs: voilà, dans quel dispositif, un perroquet, s'il est doué, pourrait apprendre à parler. La validité écologique ne porte donc plus sur les sujets de l'expérimentation, mais sur les conditions mêmes de l'expérience et seulement sur celles-ci. Ce qui veut dire qu'on se retrouve à présent avec une situation d'exception, mais cette fois, à deux niveaux : non seulement on a un être exceptionnel (tous les perroquets ne peuvent pas parler) mais surtout des dispositifs d'exception: il faut des conditions exceptionnelles, risquées, bricolées, faites d'invention, d'opportunisme et de ruse (mètis), des conditions d'exception. Le laboratoire ne représente plus la vie dehors, il n'a de portée que locale, il est un lieu exceptionnel qui peut, ou non, produire des occasions. Il est donc le lieu de l'artefact par excellence puisque sans toutes ces conditions très artéfactuelles, il est probable que le perroquet ne parle pas. Pepperberg a donc assumé, très activement assumé, que seul un artefact pouvait permettre la réussite d'un pari risqué dans un dispositif risqué - le labyrinthe n'est pas un dispositif risqué, aucune surprise n'attend l'expérimentateur, aucun réel échec non plus, à moins qu'il soit vraiment peu expérimenté : le rat n'a d'autre choix que de trouver la sortie, le succès est promis d'entrée de jeu; Alex, en revanche, pouvait ne pas être intéressé au fait de parler. 


\section{Changer de point de vue}

En tant que situations expérimentales risquées, ces dispositifs requièrent, de la part des expérimentateurs, tout un art des bonnes manières. Loin d'être une caractéristique éthique - ce qu'elle peut être toutefois dans les conséquences -, l'art des bonnes manières est un problème technique. L'art des bonnes manières n'exclut ni l'art des pièges, ni celui des leurres, pas plus qu'il ne se fonde sur un quelconque généreux principe d'égalité, de symétrie ou de réciprocité. Pepperberg, personne n'en disconviendra, fait parler son perroquet en utilisant des stratégies dont la vertu essentielle est d'être efficace. Mais elle ne cesse d'assumer que la situation créée, comme l'être qu'elle interroge, Alex, est exceptionnelle. Et elle ne cesse d'en passer, pour accomplir son projet, par la manière dont son animal peut y être intéressé, c'est-à-dire par la perspective qu'il adopte par rapport à cette situation.

Ces situations productrices de perspectives et d'intelligence sont exceptionnelles encore parce les scientifiques ont pleinement assumé le fait que produire ces intelligences, celle de l'animal et celle du scientifique, demande quantité de bonnes conditions, de l'intérêt, du tact, de l'inventivité. Je ne connais aucune situation de découverte de compétences nouvelles qui ne soit, par un aspect ou un autre, une situation qui prenne en charge de créer de l'exception au profit des possibilités de surprise: Koko qui apprend aux humains que la tristesse mélancolique n'était pas de leur seul ressort, Alex le perroquet surdoué, Ricco, le chien comprenant les mots, quantité d'animaux se bousculent à présent aux frontières des territoires de nos compétences. Quelques pies, par exemple, ont réussi dernièrement l'épreuve de la reconnaissance de soi dans un miroir ${ }^{13}$. Sur les cinq pies testées, seules deux ont pleinement réussi le test. L'échec des trois autres signe en fait la réussite du dispositif: c'est un dispositif risqué, qui s'attache à la singularité des êtres; ils peuvent réussir ou rater. Chaque réussite est d'ailleurs exceptionnelle dans la mesure même où le dispositif ne peut s'autoriser la répétition des épreuves: si celles-ci se répétaient un très grand nombre de fois, on ne pourrait exclure la possibilité de l'effet d'un conditionnement. L'exception qui caractérise l'épreuve du laboratoire remet en cause deux des contraintes auxquelles il s'est longtemps soumis: celle de la récursivité des dispositifs (les expériences doivent être répétables) et de la substituabilité des êtres, garantes de la généralisation. Ce que les labyrinthes skinnériens illustraient à merveille: tout rat doit pouvoir

13. Helmut Prior, Ariane Schwarz et Onur Güntürkün, "Mirror-Induced Behavior in the Magpie (Pici pica): Evidence of Self-Recognition", PloS Biology, 6(8) : e202. Doi: 10.1371/jour nal.pbio.0060202. 
s'inscrire dans une moyenne, reposant non sur la variété mais sur la variation. L'exception pourrait même advenir qu'elle passerait totalement inaperçue: elle se dissoudrait automatiquement dans la statistique.

Si la condition de la non-substituabilité des êtres, et donc également celle des relations «personnelles » qui se tissent entre les chercheurs et leurs animaux, semble être techniquement articulée à l'exception, elle n'en est toutefois pas une condition nécessaire. D'autres figures de l'exception, cette fois sans la condition de singularité, pourraient mettre à l'épreuve ce qui, dans ce texte, prenait subrepticement l'allure d'une généralisation. Pour cette mise à l'épreuve, la figure de Temple Grandin s'impose. Temple Grandin est une scientifique américaine travaillant depuis de longues années avec des animaux d'élevage. Elle conçoit et dessine les bâtiments dans lesquels les animaux sont amenés pour y être soignés, manipulés ou abattus, et est conseillère en ces matières. Temple Grandin est en outre autiste. Elle comprend difficilement les émotions des humains, ce qui lui fait dire qu'elle se sent parfois comme un anthropologue sur Mars ${ }^{14}$. Par ailleurs, et c'est ce qui lui permet de faire son métier, elle voit, selon ses propres termes, le "monde en images ", elle est, comme elle le dit encore, «penseur visuel», contrairement à la plupart des gens, qui sont des «penseurs verbaux». Si Temple Grandin peut faire le métier qu'elle a choisi, et le faire avec succès, c'est parce que, dit-elle, elle peut percevoir le monde tel que les vaches, dont elle est amenée à s'occuper, elles-mêmes le perçoivent. Lorsqu'elle doit résoudre un problème sur le terrain, par exemple le fait que le bétail refuse d'entrer dans un endroit où on doit l'amener fréquemment, qu'il crée des problèmes qui génèrent des conflits avec les humains qui s'en chargent, Grandin cherche à rendre lisible la manière dont les vaches voient et interprètent la situation. Le fait de comprendre ce qui pouvait effrayer l'animal, et que nous ne percevons pas, ce qui suscitait sa résistance à faire ce qu'on lui demande de faire, à entrer dans un bâtiment et à traverser un couloir, permet à Grandin de résoudre les problèmes et les conflits. Il suffit parfois d'un détail, un petit bout de chiffon coloré qui flotte sur une barrière, une tache d'ombre sur le sol, et qui ne nous apparaît pas, ou qui ne signifie pas la même chose pour nous, l'animal fait des choses incompréhensibles. Le fait qu'elle soit autiste, explique Grandin, la rend sensible aux environnements, d'une sensibilité très semblable à celle des animaux. Sa compréhension fine des animaux, sa possibilité d'adopter leur perspective, repose en fait comme sur un pari. Les animaux sont des êtres exceptionnels, comme elle-même, en tant qu'autiste, l'est.

14. C'est d'ailleurs le titre que choisira, pour un livre dont un chapitre lui est consacré, Oliver Sacks, Un anthropologue sur Mars, trad. fr. C. Cler, Paris, Le Seuil, 1996. 
L'autisme m'a doté d'une perspective sur les animaux que la plupart des professionnels n'ont pas, quoique des gens ordinaires puissent y accéder: le fait que les animaux sont plus malins que nous le pensons. [...] Les gens qui aiment les animaux et qui passent une bonne partie de leur temps avec eux, souvent commencent à sentir intuitivement qu'il y a plus pour les animaux que ce que notre regard rencontre. Mais ils ne savent pas ce que c'est, ni comment le décrire ${ }^{15}$.

Certains autistes, explique-t-elle, sont mentalement très retardés mais capables de faire des choses que les humains normaux sont incapables d'apprendre à faire, par exemple connaître le jour où vous êtes né d'après la date, en une fraction de seconde, ou encore vous dire si le numéro où vous habitez est un nombre premier. Les animaux sont comme les savants autistes.

Ils ont des talents que les gens n'ont pas de la même manière que les personnes autistes ont des talents que les gens normaux n'ont pas; certains animaux ont des formes de génie que les gens n'ont pas, de la même manière que les savants autistes ont des formes spéciales de génie $^{16}$.

Les animaux ont une capacité incroyable à percevoir des choses que les humains ne peuvent percevoir, et une capacité tout aussi incroyable de se souvenir d'informations hautement détaillées dont nous ne pourrions nous souvenir.

Je trouve cela amusant que les gens normaux disent toujours à propos des enfants autistes qu'ils vivent dans leur propre petit monde. Si vous travaillez avec des animaux, vous commencez à réaliser que vous pouvez dire exactement la même chose des gens normaux. Il y a un monde immense, magnifique autour de nous, que la plupart des normaux ne perçoivent pas $^{17}$.

Ainsi les animaux s'attachent-ils aux détails, alors que nous privilégions une vision globale parce que nous tendons à fondre ces détails dans un concept qui nous donne la perception.

\section{Des expériences qui restent singulières}

Ce que Temple Grandin met à double épreuve, ou peut-être même propose comme contre-épreuve, et qui m'intéresse, c'est l'existence de l'exception sans la singularité, d'une part; d'autre part, on y retrouve la possibilité de partager une perspective commune sans la version romantique de l'empathie.

L'exception, en effet, dans ce cadre, désigne non pas des êtres singuliers, attachés à des relations qui favorisent cette singularité, mais

15. Temple Grandin et Catherine Johnson, Animals in Translation, Orlando, Harvest Book, p. 7.

16. Ibid., p. 8.

17. Ibid., p. 24. 
des êtres qu'on pourrait désigner comme «particuliers », au sens de différents et de spéciaux. C'est une tout autre perspective qui aboutit, de fait, à entrer dans le monde de l'animal en faisant le pari d'une compréhension fondée sur le talent, sur le génie propre, et commun à certaines bêtes. Est-ce que les animaux sont comme les autistes? Grandin l'affirme avec une certitude, il est vrai, difficile à partager pour ceux qui ne sont ni animaux ni autistes. Mais le régime de vérité qui accompagne cette affirmation relève bien du pragmatisme; en se comportant comme si elle avait affaire à des êtres qui, comme elle, voient le monde d'une certaine façon, ont le génie du détail et le talent de la perception, elle arrive à obtenir de ces êtres ce qui faisait l'objet de ce pari : accorder mieux des intentions, celles des éleveurs et celles des animaux.

Par ailleurs, Grandin sort la question «perspectiviste», le fait de pouvoir se mettre à la place d'un autre pour comprendre ses peurs et ses intentions, de la sphère à laquelle cette question est généralement arraisonnée: celle des sentiments et de l'empathie. Certes, on a bien affaire à une forme d'empathie, mais une empathie sans pathos. Il est difficile de donner un nom à ce qui relèverait de ce type d'oxymore: empathie sans pathos. C'est une forme d'agencement qui certes implique des corps, puisqu'elle écrit:

La première chose que je fais systématiquement, parce que nous ne pouvons résoudre un mystère animal à moins de nous mettre à sa place - littéralement à leur place -, c'est d'aller là où l'animal va et faire ce que l'animal fait ${ }^{18}$.

Mais c'est une forme d'empathie technique, qui ne se fonde pas sur un partage d'émotions, plutôt sur la création d'une communauté de sensibilité visuelle, sur un talent bien plus cognitif qu'émotif, puisque c'est ainsi que nous catégorisons ce type de processus. Si je trouve peu de mots pour rendre compte de cet événement, moi qui m'inscris dans une tradition pour laquelle l'empathie relève de la sphère des émotions partagées, je peux néanmoins renvoyer à cette petite merveille expérimentale qu'est le roman de science-fiction, le Paidhi, de Carolyn Janice Cherryh ${ }^{19}$. Dans un univers lointain de nous tant dans le temps que dans l'espace, un ambassadeur terrien est envoyé sur une planète où d'étranges êtres, très semblables à nous, vivent, entrent en relation, parlent, et tentent de résoudre des conflits. Or, c'est ce qui les rend étranges, les êtres de cette planète ne connaissent aucune émotion. Il n'y a entre eux ni amour, ni amitié, ni haine, ni affect. Et toute la difficulté de l'ambassadeur humain est de comprendre un système relationnel à ce point similaire au nôtre,

18. T. Grandin, Animal in Translation, op. cit., p. 31.

19. Carolyn Janice Cherryh, le Paidhi, Paris, J'ai lu, 1998. Le titre original du livre en anglais était Foreigners. 
où les gens s'entraident ou se trucident, cultivent des liens, alors que lui-même est toujours tenté de le traduire en termes émotionnels ${ }^{20}$. Ce qui tient les gens, ce qui les lie et ce qui explique leurs conduites se fonde en fait sur des relations d'allégeances et de loyautés, qui prescrivent, comme un ensemble de règles, les codes de conduite. Et cela produit un type de société et de relations à ce point semblables aux nôtres que le héros ne cesse de se méprendre sur les motifs et les intentions de ceux qui l'aident ou se comportent en ennemis.

C'est bien à une véritable expérimentation que nous sommes conviés, celle de comprendre à quel point nos affects peuvent être lus comme un ensemble de codes et de scripts qui nous permettent d'entrer en relations, quoiqu'elles leur donnent, il est vrai, une touche inoubliable, une «importance» de premier ordre. C'est ainsi, en nous touchant sur ce mode, avec une efficacité qu'on aurait pu penser inégalée, que d'autres êtres en viennent à importer pour nous. Ce qui m'intéresse dans ce rapprochement qui m'est offert par le roman, c'est de penser des situations d'attachement et d'exception, comme Grandin nous les décrit, sur d'autres modes que ceux qui nous sont familiers. Car les termes de scripts et de codes pour désigner les émotions ressemblent, à l'en croire, à son expérience avec les humains, qu'elle ne peut comprendre qu'à l'aide de scénarios mémorisés qui traduisent, sur un autre mode, leurs motifs et leurs émotions ${ }^{21}$.

Nous sommes cependant, en dernier ressort, renvoyés à la singularité, par le biais de l'exception. Car ces situations que décrit Temple Grandin sont sans conteste non seulement des situations d'exception mais également et surtout des situations à haute teneur en singularité : être autiste et posséder ce talent n'a rien d'une situation généralisable. C'est une chance, un kairos diraient les Grecs anciens, un événement singulier qui fait occasion.

Et c'est sans doute là ce qui lie, d'un fil commun, toutes ces situations que nous avons évoquées, toutes ces recherches qui ont rendu les animaux plus intéressants: ce sont des situations risquées, dont nous savons qu'elles reposent certes sur la chance, mais une chance qu'il nous faut apprendre à apprivoiser.

\section{Vinciane Despret}

20. On trouvera ce type d'expérience dans le témoignage que rapporte l'anthropologue Monique Jeudy Ballini de ses séjours chez les Sulka de Papouasie: lorsqu'elle demande à son informateur le plus proche, avec qui elle a noué une relation privilégiée, comment il explique leur amitié, elle s'entend répondre qu'il n'y a pas d'amitié entre eux, et que ce sont des foutaises inventées par les Blancs. "Voir et regarder», Gradhiva, 1994, 15, p. 59-74.

21. Voir O. Sacks, Un anthropologue sur Mars, op. cit. 